\title{
Dynamic inversion control of UAV using ANN
}

\author{
Gong-Cai Xin ${ }^{\dagger}$ and Wei-Lun Chen \\ Department of Instrument and Electric Engineering \\ The First Aeronautical Institute of Air Force \\ Xinyang, Henan, China \\ E-mail:xingc2752@163.com
}

\begin{abstract}
The paper proposes a method to design ANN dynamic inversion controller through online ANN compensating inversion error. It mainly aims at evident shortage of dynamic inversion controller of UAV. A single hidden layer ANN structure is constructed and the stability of the whole closed loop system is proved. Also the stable adjustment arithmetic of online ANN weight is proposed. The robustness, the adaptability to fault and the response capability to actuator delay time of the scheme are verified by simulation. It is also proved that the online ANN has improved the performance of dynamic inversion controller well. It has important reference value for designing advanced flight control systems of UAV.

Keywords: Dynamic Inversion Controller; Unmanned Aerial Vehicle (UAV); Neural Network (ANN); Single Hidden Layer (SHL).
\end{abstract}

\section{Introduction}

The speciality on using ANN to approach random nonlinear function has already become a kind of latent settle method to many outstanding problems about nonlinear adaptive system and robustness control system. At present, the foreign countries do a mass of researches on designing flight control system by ANN. Two kinds of system info have great influence: one is ANN reference model based adaptive control, it roots from Narendra's classical literature ${ }^{[1]}$; the other is based on ANN dynamic inversion control, it is applied in tactics missile, UAV, unmanned helicopter and rotorcraft ${ }^{[2]}$. This paper studies UAV based on ANN dynamic inversion control. On line ANN is introduced on the gesture control loop of UAV's dynamic inversion controller. It is used to compensate inverse error caused by all kinds of disturbances and improve the dynamic inversion controller's performance. 


\section{Design of ANN Dynamic Inversion}

When we design UAV's nonlinear dynamic inversion controller by singular perturbation theory and dynamic inversion method, the primary state variables are taken as rate of roll $\mathrm{p}$, rate of pitch q, rate of yaw $\mathrm{r}$, roll angle $\phi$, pitch angle $\theta$, yaw angle $\psi$, angle of attack $\alpha$, angle of sideslip $\beta$ and airspeed $\mathrm{V}$; and the primary control variables are elevator deflection angle $\delta$ e, aileron deflection angle $\delta a$, rudder deflection angle $\delta$. According to the movement rule of UAV, the state variables are divided into quick variables $(\mathrm{p}, \mathrm{q}, \mathrm{r})$, relatively slow variables $(\phi, \theta, \psi)$ and slow variables $(\mathrm{V}, \alpha, \beta)$. The nonlinear equation group is decomposed into three different time scale subsystems. And the relatively slow subsystem of UAV, that is, gesture subsystem is described as

$$
\dot{X}_{2}=f\left(X_{1}, X_{2}, \delta\right)
$$

Where,

$$
X_{1}=[p, q, r]^{T}, X_{2}=[\phi, \theta, \psi]^{T}, \delta=\left[\delta_{e}, \delta_{\alpha}, \delta_{r}\right]^{T}
$$

If the result for counteracting inversion controller is accurate, we can define pseudo-control variable as

$$
u=X_{2}=f\left(X_{1}, X_{2}, \delta\right)
$$

Because the inversion is not accurate, the counteracting error exists. The inversion error is

$$
\dot{X}_{2}=u+\Delta^{\prime}\left(X_{1}, X_{2}, u\right)
$$

Where,

$$
\Delta^{\prime}\left(X_{1}, X_{2}, u\right)=f\left(X_{1}, X_{2}, \hat{\delta}\right)-f\left(X_{1}, X_{2}, \delta\right)
$$

To compensate the inversion error by online ANN (shown in fig.1), the control structure of the ith chaANNel is

$$
u_{i}=u_{p i}+\dot{x}_{2 c i}-\dot{u}_{a d i}, \quad i=1,2,3
$$

Where, $\hat{u}_{\text {adi }}$ is a adaptive item and is used to compensate inversion error, it is realized by ANN; ${ }_{p i}$ is proportional control item and is represented by

$$
u_{p i}=k_{p i}\left(x_{2 c i}-x_{2 i}\right)
$$

By (3),(5),(6), we can get 


$$
u_{i}=\dot{x}_{2 i}-\Delta_{i}^{\prime}=k_{p i}\left(x_{2 c i}-x_{2 i}\right)+\dot{x}_{2 c i}-\hat{u}_{a d i}
$$

Let

$$
e_{i}=\tilde{x}_{2 i}=x_{2 c i}-x_{2 i}, \dot{e}_{i}=\dot{x}_{2 c i}-\dot{x}_{2 i}
$$

then

$$
\dot{e}_{i}=-k_{p i} e_{i}+\left(\hat{u}_{a d i}-\Delta_{i}^{\prime}\right)
$$

By the above equations, we know, if the adaptive item can reconfigure inversion error in the ideal condition, the system error ei will be approach to zero gradually.
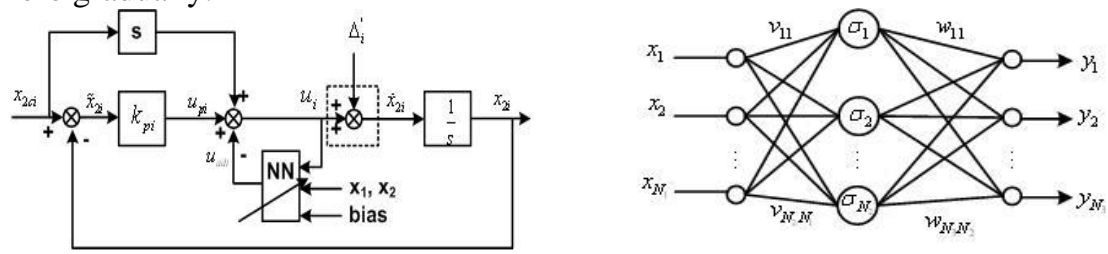

Fig.1. The principle of dynamic inversion control Fig. 2.The structure of SHL ANNof ANN

\subsection{The structure of online ANN}

SHL ANN is a full variable approximate controller. If the scale of network is big enough (of course, the scale can't be oversize in view of realization and executing efficiency), it can approach nonlinear function by any precision. The paper introduced a method to compensate inversion error by a SHL online ANN (shown as fig.2).

Stimulant function $\sigma(\cdot)$ is defined as sigmoid function

$$
\sigma(z)=\frac{1}{1+e^{-a z}}
$$

Where, $z \in R, a>0$ is stimulant depth, the slope of control function. Let input vector $\bar{X}=\left[\begin{array}{llll}1 & x_{1} & \cdots & \left.x_{N_{1}}\right]^{T}\end{array}\right.$, the output vector $y=\left[\begin{array}{llll}y_{1} & y_{2} & \cdots & y_{N_{3}}\end{array}\right]^{T}$. The threshold value vector $\left[\begin{array}{llllll}\theta_{v 1} & \theta_{v 2} & \cdots & \theta_{v N_{2}}\end{array}\right]^{T},\left[\begin{array}{lllll}\theta_{w 1} & \theta_{w 2} & \cdots & \theta_{w N_{3}}\end{array}\right]^{T}$ is included in the first column of weighted matrix VT and WT respectively

$$
V^{T}=\left[\begin{array}{cccc}
\theta_{v 1} & v_{11} & \cdots & v_{1 N_{1}} \\
\theta_{v 2} & v_{21} & \cdots & v_{2 N_{1}} \\
\vdots & \vdots & \vdots & \vdots \\
\theta_{v N_{2}} & v_{N_{2} 1} & \cdots & v_{N_{2} N_{1}}
\end{array}\right] \quad W^{T}=\left[\begin{array}{cccc}
\theta_{w 1} & w_{11} & \cdots & w_{1 N_{2}} \\
\theta_{w 2} & w_{21} & \cdots & w_{2 N_{2}} \\
\vdots & \vdots & \vdots & \vdots \\
\theta_{w N_{3}} & w_{N_{3} 1} & \cdots & w_{N_{3} N_{2}}
\end{array}\right]
$$


Therefore, the mapping of SHL ANN can be written as matrix as follow

$$
y_{i}=W_{i}^{T} \sigma\left(V^{T} \bar{X}\right)=W^{T} \sigma\left(V^{T} \bar{X}\right), \quad i=1,2, \cdots, N_{3}
$$

Where $W_{i}^{T}=W^{T}$ represents the ith column of matrix WT.

In fig.2, by SHL network structure and SHL network full approximation theory, to continuous uncertain nonlinear inversion error function $\Delta_{i}^{\prime}$ and any given inversion reconfigurable error $\varepsilon_{N}$, there exist finite hidden nerve cells N2 and weighted matrix $\mathrm{V}, \mathrm{W}$,

$$
\Delta_{i}^{\prime}=W^{T} \sigma\left(V^{T} \bar{X}\right)+\varepsilon,\|\varepsilon\| \leq \varepsilon_{\mathrm{N}}
$$

Let adaptive item

$$
\hat{u}_{\text {adi }}=\hat{W}^{T} \sigma\left(\hat{V}^{T} \bar{X}\right)
$$

Where, $\hat{W}^{T}, \hat{V}^{T}$ is respectively hopeful weighted value of $W^{T}, V^{T}$, take control structure

$$
u_{i}=u_{p i}+\dot{x}_{2 c}+\bar{v}-\hat{u}_{a d i}, \quad i=1,2,3
$$

Where, $\bar{v}$ is robustness item. Then the system dynamic error can be written as

$$
\dot{e}_{i}=-k_{p i} e_{i}+\left(\hat{W}^{T} \sigma\left(\hat{V}^{T} \bar{X}\right)-W^{T} \sigma\left(V^{T} \bar{X}\right)\right)-\bar{v}-\varepsilon
$$

\subsection{Prove the closed-loop stability of controller}

To prove the closed-loop stability of ANN dynamic inversion controller by Lyapunov stability theory. Let the Lyapunov function

$$
U=\frac{1}{2} e_{i}^{2}+\operatorname{tr}\left(\tilde{W}^{T} F^{-1} \tilde{W}\right)+\operatorname{tr}\left(\tilde{V}^{T} G^{-1} \tilde{V}\right)
$$

Where, F-1 and G-1 are the symmetrical positive matrix with suitable dimension; $\tilde{V}=\hat{V}-V$ and $\tilde{W}=\hat{W}-W$ are respectively the error variables of weighted matrix $\mathrm{V}$ and $\mathrm{W}$.

In order to analyze stability, take derivative on both sides of (15), we have

$$
\begin{aligned}
& \dot{U}=e_{i} \dot{e}_{i}+\operatorname{tr}\left(\tilde{W}^{T} F^{-1} \dot{\tilde{W}}\right)+\operatorname{tr}\left(\tilde{V}^{T} G^{-1} \dot{\tilde{V}}\right) \\
& \left.=e_{i}\left[-k_{p i} e_{i}+\hat{W}^{T} \sigma\left(\hat{V}^{T} \bar{X}\right)-W^{T} \sigma\left(V^{T} \bar{X}\right)\right)-\bar{v}-\varepsilon\right]+\operatorname{tr}\left(\tilde{W}^{T} F^{-1} \dot{\tilde{W}}\right)+\operatorname{tr}\left(\tilde{V}^{T} G^{-1} \dot{\tilde{V}}\right)
\end{aligned}
$$

By literature [1], if choose 


$$
\begin{array}{r}
\dot{v}=k_{v}\left(\|\hat{Z}\|_{F}+Z_{M}\right) e_{i} \\
\dot{\hat{W}}=F \hat{\sigma} e_{i}-F \hat{\sigma}^{\prime} \hat{V} \bar{X} e_{i}-k_{w} F\left\|e_{i}\right\| \hat{W} \\
\dot{\hat{V}}=G \bar{X} \hat{W}^{T} \hat{\sigma}^{\prime} e_{i}-k_{v} G\left\|e_{i}\right\| \breve{V}
\end{array}
$$

We can get $\dot{U}<0$

That is the derivative of Lyapunov function is negative matrix. So the system error and the astringency of weight have proved.

\subsection{Simulation and discussion of cases}

Here are many simulations for UAV ANN dynamic inversion controller according to the above design. Here are several cases. The initial condition of simulation: the mass of UAV $\mathrm{m} 0=13.5 \mathrm{~kg}$, height above sea level $\mathrm{h} 0=1000 \mathrm{~m}$, airspeed $\mathrm{V} 0=25(\mathrm{~m} / \mathrm{s})$, pitch angle $\theta 0=-5^{\circ}$, the initial time $\mathrm{t}=0 \mathrm{~s}$, input pitch angle $\theta \mathrm{c}=10^{\circ}$, the number of ANN hidden unit is 10 , the length of simulation step is $10 \mathrm{~ms}$. From fig.3 to fig.5, the $\mathrm{x}$-axis is time $\mathrm{t} / \mathrm{s}$, the $\mathrm{y}$-axis is respectively pitch angle $\theta\left(^{\circ}\right)$, rate of pitch $\mathrm{q}\left({ }^{\circ}\right) / \mathrm{s}$, yaw angle of elevator $\delta \mathrm{e}\left({ }^{\circ}\right)$, airspeed $\mathrm{V}(\mathrm{m} / \mathrm{s})$, angle of attack $\alpha\left(^{\circ}\right)$ and error of pitch angle e $\left(^{\circ}\right)$.

Case 1: Due to the restriction of actuator, the range of location and velocity are respectively $-32^{\circ} \sim 32^{\circ}$ and $-30\left(^{\circ}\right) / \mathrm{s} \sim 30\left(^{\circ}\right) / \mathrm{s}$. The aim is to simulate the longitudinal motion of UAV.

Fig.3 shows that the astringent velocity of online ANN is rapidly and the response of control system is quick and the system has strong robustness.

Case 2: Fault simulation, to validate the reconfigurable ability for ANN to fault. Suppose the default of elevator is $50 \%$ in restrictive condition in simulation 1 when the simulation runs at 20s. Fig.4 shows that the fault of rudder surface makes the controlled object model inaccurate. The broken line represents control system can not make the controlled objects run after the hopeful output if there is no online ANN modification; or else, the system can control accurately and run after the output, represented by real line. Here, the stable value of yaw angle of elevator is one times of no default and it can overcome the effect of actuator default. We can see, ANN dynamic inversion control system has strong reconfigurable ability.

Case 3 In the restriction condition of simulation 1 , delay 0.2 s to dynamic states of actuator which model is not be founded. 
Fig.5 shows that the scheme of this paper is more sensitive to dynamic actuator which is not be modeled. And the work states of controlled system have very strong oscillation, the response of pitch angle becomes worsen, and the saturation state of actuator switches quickly and frequently, it is terrible. If only delay $0.1 \mathrm{~s}$, the weight will be convergent in online updating, the oscillation of work state will be avoided and pitch angle is convergent. So the delay time of actuator should be small as possible when we design controller.
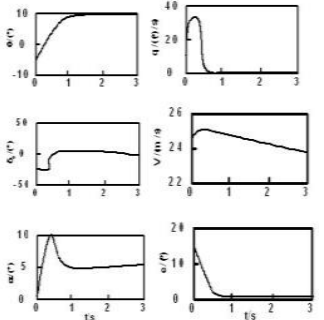

Fig.3. Longitudinal motion of UAV by ANN dynamic inversion controller
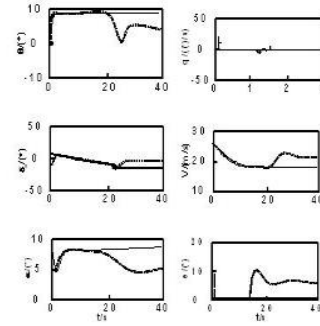

Fig.4. The default $50 \%$

of elevator at $20 \mathrm{~s}$ (the real line is

ANN, the broken line is no ANN)
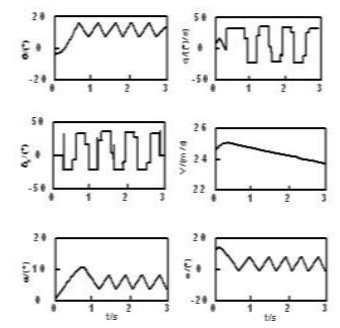

Fig.5. Delay time(0.2s)

of actuator controlled by ANN dynamic inversion

\section{Conclusion}

This paper affords a design scheme about UAV dynamic inversion controller based on online ANN. By theory analysis and simulation, this scheme has good control effect. Dynamic inversion is realized easily and we can do simplification and approximation to model in the course of getting inversion. The error caused by this way compensated by online iterative ANN. This control scheme acts compensation to modeling imprecise in restriction, fault of actuator of UAV; but it is sensitive to the inversion error caused by non modeling (with delay time). So we should make the delay time as small as possible in the course of designing controller. The next work is to deal with non modeling dynamic states system.

\section{References}

1. Narendra K S, Parthasarathy K: Identification and Control of Dynamical System Using Neural Networks [J]. IEEE Transactions on Neural Networks, (1990) 1 ( 1) : 4-27.

2. Kim B S, Calise A J: Nonlinear Flight Control Using Neural Networks [J]. Journal of Guidance Control and Dynamics, (1997) 20 (1): 26-33.

3. Lewis F L, Yesildirek A, Kai L: Multilayer Neuralnet Robot Controller with Guaranteed Tracking Performance [J]. IEEE Transactions on Neural Networks, (1996) 7 (2): 388-399. 